\title{
ISYDE- $\Psi^{*}$ Prima fase dell'implementazione delle linee guida per le attività di psicologia in cardiologia riabilitativa e preventiva
}

*Italian SurveY on CarDiac REhabilitation - Psychology

\section{ISYDE- $\Psi^{*}$ First step of the implementation of guidelines for psychology activities in cardiac rehabilitation and prevention *Italian SurveY on CarDiac REhabilitation - Psychology}

\author{
Marinella Sommaruga, Roberto Tramarin (Coordinatori), Gianluigi Balestroni, \\ Ornella Bettinardi, Maria Antonella Cauteruccio, Massimo Miglioretti, \\ Marco Monti, Ombretta Omodeo, Antonia Pierobon, Paola Vaghi
}

\begin{abstract}
ISYDE- $\Psi^{*}$ First step of the implementation of guidelines for psychology activities in cardiac rehabilitation and prevention. *Italian SurveY on CarDiac REhabilitation - Psychology. M. Sommaruga, R. Tramarin (Coordinatori), G. Balestroni, O. Bettinardi, M.A. Cauteruccio, M. Miglioretti, M. Monti, O. Omodeo, A. Pierobon, P. Vaghi.

The Italian SurveY on CarDiac REhabilitation - Psychology (ISYDE- $\Psi$ ) was developed, in line with the indications of the Italian National Guidelines Program, as part of a project to implement and apply the guidelines for psychology interventions in cardiac rehabilitation and prevention published in 2003. The Task Force on Psychological Interventions in Cardiac Rehabilitation conducted this pilot survey of the existing situation of Psychology in order to prepare the ground for implementation of the guidelines through interactive training. As part of the evaluation of
\end{abstract}

training requirements a questionnaire was elaborated to gather information on the models of organization of and activities carried out by psychologists working in the surveyed cardiac rehabilitation facilities. Data collection for ISYDE- $\Psi$ terminated at the end of March 2005, with replies from $68 / 107(63.6 \%)$ structures. In the light of this response, the Task Force has developed a training project for psychologists working in cardiac rehabilitation, sponsored by the Italian Council of Psychologists, that will be implemented in different regions of the country with the aim of disseminating the guidelines and promoting their correct application despite the existing regional disparities in organization.

Keywords: psychology, cardiac rehabilitation, guidelines.

Monaldi Arch Chest Dis 2005; 64: 53-58.

Gruppo di Lavoro della Task Force sull'intervento psicologico in cardiologia riabilitativa del Gruppo Italiano di Cardiologia Riabilitativa e Preventiva.

Corresponding author: Dr.ssa Marinella Sommaruga; Servizio di Psicologia; IRCCS Fondazione Salvatore Maugeri; Via Roncaccio, 16 - I-21049Tradate(VA), Italy; E-mail address: msommaruga@fsm.it

In occasione del VI Congresso Nazionale del Gruppo Italiano di Cardiologia Riabilitativa e Preventiva (GICR) che si è svolto a Cosenza nell'ottobre 2002 è stata presentata la prima elaborazione dei dati del Censimento Italiano delle Strutture di Cardiologia Riabilitativa in Italia e dello Studio ISYDE (ISYDE - Italian SurveY on carDiac rEhabilitation) inerente all'analisi dei percorsi riabilitativi del paziente con esiti di infarto del miocardio e di cardiochirurgia. ${ }^{1}$ Già in questo primo censimento emergevano una serie di dati relativi alle attività di psicologia in cardiologia riabilitativa; tuttavia il carattere di ricognizione di questa prima raccolta dati non consentiva una accurata valutazione della effettiva presenza dello psicologo nei singoli programmi riabilitativi, del contenuto del suo intervento e degli specifici aspetti di tipo organizzativo. Nello stesso Congresso veniva presentato l'avanzamento dei lavori (iniziati nel maggio 2001) della Task Force per le Attività di Psicologia in Cardiologia Riabilitativa e Preventiva, cui era stato affidato dal Direttivo del GICR il compito di definire lo stato dell'arte dell'intervento psicologico in cardiologia riabilitativa sulla base delle prove di efficacia, e che avrebbe portato alla pubblicazione delle Linee Guida per le Attività di Psicologia Riabilitativa e Preventiva. ${ }^{2}$

Con la finalità di valutare la conoscenza e la diffusione delle linee-guida, per promuoverne e favorime la diffusione, l'implementazione e di ottenere informazioni sempre aggiornate sulle modalità organizzative ed operative dell'intervento psicologico la Task Force ha avviato una indagine co- 
noscitiva che permettesse di aggiornare e integrare lo studio ISYDE.

L'indagine conoscitiva ISYDE- $\Psi$ nasce dunque, in coerenza con le indicazioni del Piano Nazionale Linee Guida, ${ }^{3}$ all'interno di un progetto di implementazione ed applicabilità delle linee guida per le attività di psicologia in cardiologia riabilitativa e preventiva pubblicate e ne costituisce una prima fase.

E noto che il termine implementazione indica l'introduzione nella pratica corrente delle linee guida utilizzando strategie di intervento appropriate, atte cioè a favorirne l'utilizzo e a rimuovere $\mathrm{i}$ fattori di ostacolo al cambiamento. ${ }^{3}$ Alcuni fattori sono relativi alle barriere esterne (mancanza di risorse, vincoli organizzativi, mancanza di incentivi, tempi stretti), altri riguardano gli atteggiamenti e il comportamento dei singoli operatori (inerzia, incapacità a cambiare le abitudini consolidate da anni di pratica professionale). La letteratura indica un buon numero di strategie di intervento efficaci: formazione attraverso visite di facilitazione esterna (outreach visits), interventi multipli di auditing e feedback, processi di consenso locali, marketing ed incontri formativi interattivi.

Anzitutto è necessario comprendere i fattori in grado di influenzare il processo di cambiamento, coinvolgendo il maggior numero possibile di potenziali utilizzatori e beneficiari delle linee guida. In questa prospettiva il Gruppo di Lavoro della Task Force nel marzo 2004 ha identificato una prima fase di valutazione del contesto per rendere possibile una ipotesi di reale implementazione attraverso programmi di formazione che siano il più possibile interattivi, $4-10$ articolata nei seguenti momenti: 1. Ideazione e costruzione di un questionario (figura 1) per raccogliere informazioni dettagliate sulle attività di psicologia già presenti nelle strutture riabilitative censite e orientato a rilevare le differenti strategie organizzative, logistiche, di valutazione e d'intervento esistenti fra gli psicologi operanti nell' ambito della riabilitazione cardiologica.

2. invio, nel giugno del 2004 via e-mail e posta convenzionale, di una lettera di presentazione con il razionale e gli obiettivi dello studio e del questionario ai responsabili cardiologi e agli psicologi di tutte le strutture di cardiologia riabilitativa già identificate dal censimento ISYDE-2001. Dal primo censimento ISYDE-2001 erano state individuate 156 strutture di cui 88 con indicazione del nome di uno psicologo referente. Alla fine del mese di novembre sono pervenuti 58 degli 88 questionari inviati. I dati preliminari relativi a queste 58 strutture sono stati presentati al VII Congresso Nazionale del GICR nell'ottobre del 2004.

3. nel novembre 2004, alle 88 strutture individuate dal Censimento ISYDE-2001 se ne sono aggiunte altre 19 che nel frattempo avevano intrapreso una attività psicologica strutturata, derivate dall'aggiornamento ISYDE-2004. A queste strutture è stato inviato il questionario e, contestualmente, si è proceduto a reiterare le richieste di aggiornamento dei dati alle strutture dalle quali non era pervenuta alcuna risposta, attra- verso contatti telefonici, e-mail, fax e posta convenzionale.

La raccolta dati si è conclusa il 31 marzo 2005, con la risposta di $68(63.6 \%)$ di 107 strutture (Appendice). Nella tabella 1 è riportato il numero di strutture suddiviso per Regione.

Quantunque l'analisi complessiva dei dati dell'indagine sia ancora in corso, sono possibili alcune prime considerazioni.

La percentuale delle risposte ottenute si attesta su valori relativamente bassi che rendono certamente complesso il tentativo sia di verificare la conoscenza e l'implementazione delle linee guida, sia di comprendere la tipologia e l'organizzazione del lavoro psicologico che viene attuato in cardiologia riabilitativa.

Come fase successiva e anche alla luce di queste considerazioni la Task Force ha sviluppato una proposta formativa, recentemente approvata dal Consiglio Nazionale dell'Ordine degli Psicologi che patrocinia e finanzia l'iniziativa. Questa proposta ha l'obiettivo di diffondere tra gli psicologi le "Linee Guida per le attività di psicologia in cardiologia riabilitativa e preventiva" in quanto documento che definisce $\mathrm{i}$ percorsi diagnostici e terapeutici ed illustra in maniera sistematica standard, opzioni e raccomandazioni per una buona pratica dell'intervento psicologico in cardiologia riabilitativa. In questo contesto sono in fase di organizzazione una serie di eventi formativi interattivi che avranno luogo in differenti ambiti territoriali nazionali.

\section{Riassunto}

L'indagine conoscitiva ISYDE- $\Psi$ (Italian SurveY on CarDiac REhabilitation - Psychology) nasce, in coerenza con le indicazioni del Piano Nazionale Linee Guida, all'interno di un progetto di implementazione ed applicabilità delle linee guida italiane per le attività di psicologia in cardiologia riabilitativa e preventiva pubblicate nel 2003. Il Gruppo di Lavoro della Task Force sull' intervento psicologico in cardiologia riabilitativa ha effettuato quest'indagine conoscitivo-esplorativa dei contesti psicologici esistenti per consentire una reale implementazione attraverso percorsi formativi interattivi. L'indagine dei bisogni formativi ha previsto l'elaborazione di un questionario atto ad indagare le tipologie organizzative e le attività svolte dagli psicologi presenti nelle 107 strutture riabilitative censite. La raccolta dati ISYDE- $\Psi$ si è conclusa alla fine di marzo 2005, con la risposta di $68(63.6 \%)$ di 107 strutture riabilitative censite. Alla luce anche delle risposte giunte, la Task Force ha sviluppato un progetto formativo, destinato agli psicologi che operano in cardiologia riabilitativa, patrocinato e sostenuto dall'Ordine degli Psicologi, che avrà luogo in differenti ambiti territoriali nazionali al fine di disseminare le linee guida e di favorirne una corretta applicazione nonostante le diverse ed eterogenee realtà organizzative esistenti.

Parole chiave: psicologia, cardiologia riabilitativa, linee guida, censimento. 


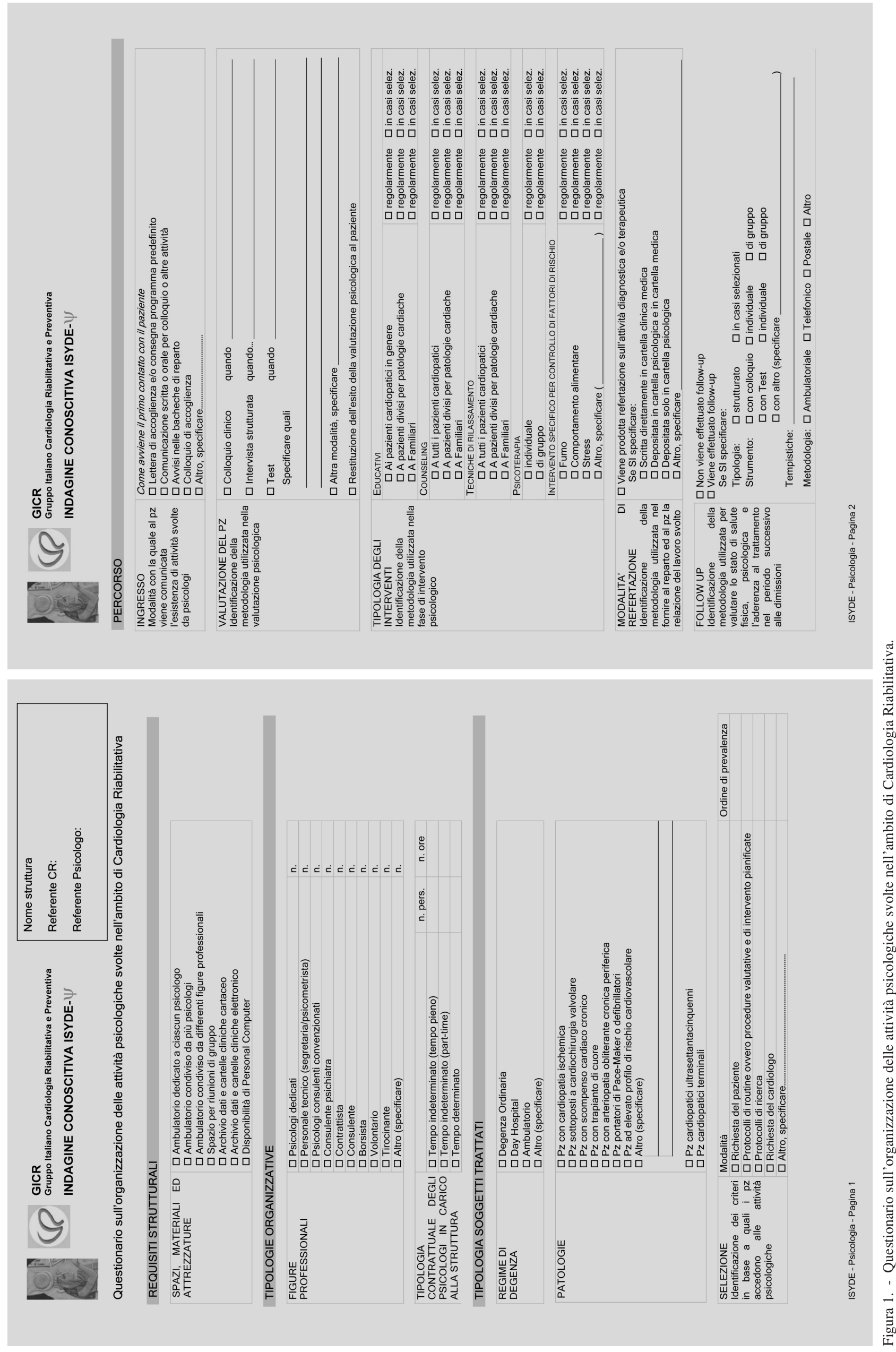



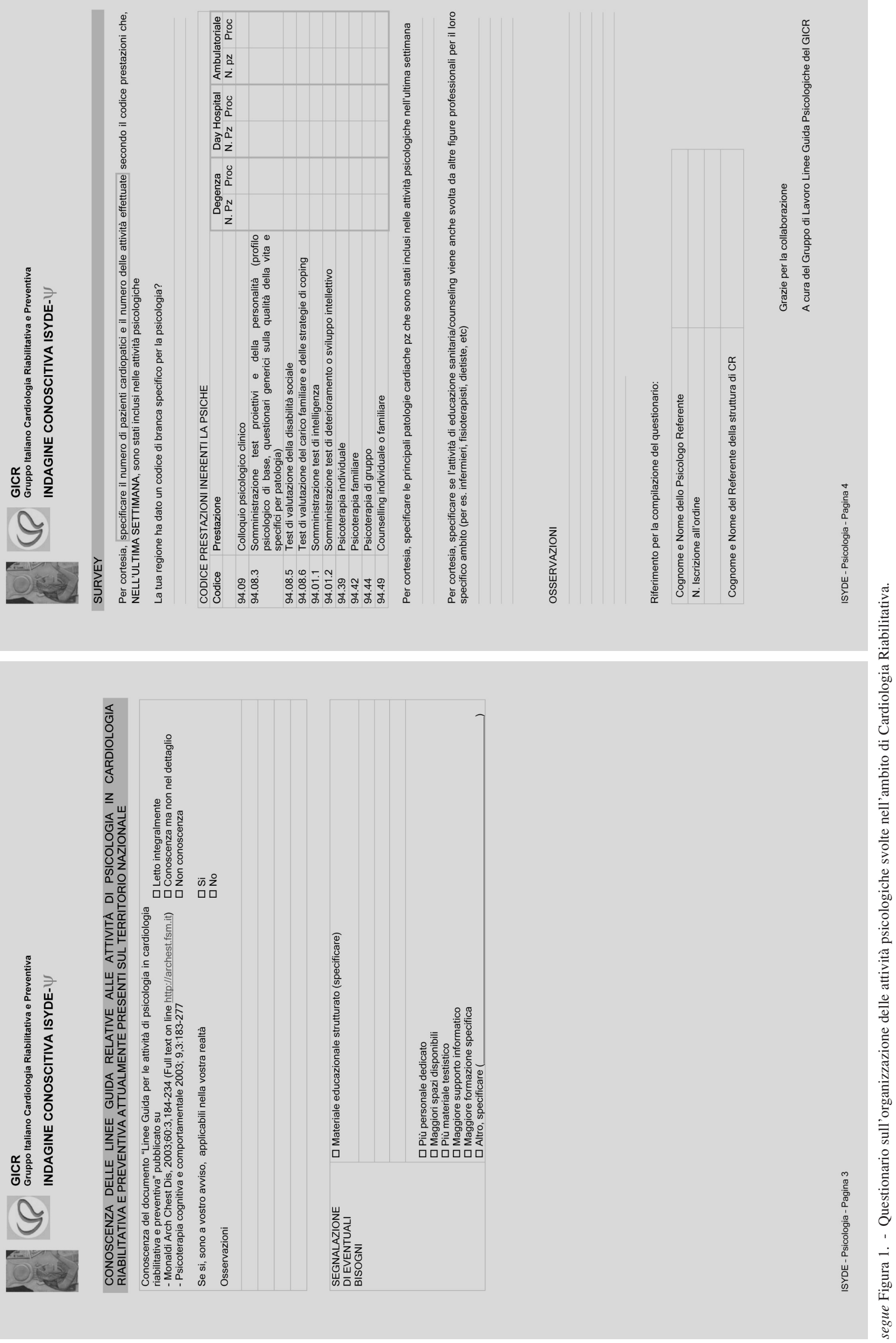
Tabella 1 - Dettaglio del numero di strutture partecipanti allo studio ISYDE- $\Psi$, suddiviso per Regione

\begin{tabular}{|c|c|c|c|}
\hline & $\begin{array}{l}\text { N. strutture } \\
\text { partecipanti } \\
\text { ISYDE- } \Psi\end{array}$ & $\begin{array}{l}\text { N. strutture } \\
\text { censite }\end{array}$ & $\%$ risposte \\
\hline Abruzzo & 1 & 1 & 100.0 \\
\hline Basilicata & 1 & 1 & 100.0 \\
\hline Calabria & 1 & 8 & 12.5 \\
\hline Campania & 5 & 8 & 62.5 \\
\hline Emilia & 5 & 7 & 71.4 \\
\hline Friuli & 2 & 6 & 33.3 \\
\hline Lazio & 2 & 3 & 66.7 \\
\hline Liguria & 2 & 2 & 100.0 \\
\hline Lombardia & 16 & 21 & 76.2 \\
\hline Marche & 0 & 0 & 0.0 \\
\hline Piemonte & 4 & 5 & 80.0 \\
\hline Trentino & 3 & 4 & 75.0 \\
\hline Puglia & 3 & 6 & 50.0 \\
\hline Sardegna & 0 & 1 & 0.0 \\
\hline Sicilia & 3 & 7 & 42.9 \\
\hline Toscana & 7 & 9 & 77.8 \\
\hline Umbria & 3 & 4 & 75.0 \\
\hline Valle Aosta & 0 & 1 & 0.0 \\
\hline Veneto & 10 & 13 & 76.9 \\
\hline Totale & 68 & 107 & 63.6 \\
\hline
\end{tabular}

\section{Bibliografia}

1. Urbinati S, Fattirolli F, Tramarin R, Chieffo C, Temporelli P, Griffo R, Belardinelli R, Vaghi P, Briolotti L. Gruppo Italiano di Cardiologia Riabilitativa e Preventiva The ISYDE project. A survey on Cardiac Rehabilitation in Italy. Monaldi Arch Chest Dis 200360 (1): 16-24.

2. Task Force per le Attività di Psicologia in Cardiologia Riabilitativa e Preventiva, Gruppo Italiano di Cardiologia Riabilitativa e Preventiva (GICR). Linee guida per le attività di psicologia in cardiologia riabilitativa e preventiva. Monaldi Arch Chest Dis 200360 (3): 184-234.

3. PNLG. Come produrre, diffondere e aggiornare raccomandazioni per la pratica clinica, Manuale metodologico. Milano: Arti Grafiche Passoni, 2002.

4. Partridge MR. Translating research into practice: how are guidelines implemented? Eur Respir J Suppl 2003 Jan; 39: 23s-29s.

5. Burgers JS, Grol RP, Zaat JO, Spies TH, van der Bij AK, Mokkink HG. Characteristics of effective clinical guide- lines for general practice. Br J Gen Pract 2003 Jan; 53 (486): $15-9$.

6. Formoso G, Liberati A, Magrini N. Survey of Italian physicians by professional setting. Arch Intern Med 2001 Sep 10; 161 (16): 2037-42.

7. Silagy CA, Weller DP, Lapsley H, Middleton P, ShelbyJames T, Fazekas B. The effectiveness of local adaptation of nationally produced clinical practice guidelines. Fam Pract 2002 Jun; 19 (3): 223-30.

8. Gross PA Implementing evidence-based recommendations for health care: a roundtable comparing European and American experiences. J Qual Improv 2000 Sep; 26 (9): 547-53.

9. Dopson S, Locock L, Chambers D, Gabbay J. Implementation of evidence-based medicine: evaluation of the Promoting Action on Clinical Effectiveness programme. $J$ Health Serv Res Policy 2001 Jan; 6 (1): 23-31.

10. Michie S, Johnston M Changing clinical behaviour by making guidelines specific. BMJ $2004 \mathrm{Feb} 7 ; 328$ (7435): 343-5. 
Appendice 1. - Elenco delle strutture che hanno aderito allo studio ISYDE- $\Psi$

\section{STRUTTURA DI CARDIOLOGIA RIABILITATIVA}

Centro di Riabilitazione Cardiologica Ospedale di Ala ,UO di Cardiologia

Casa di Cura Eremo

Ospedale San Pancrazio, Sezione di Riabilitazione Cardiologica

ASL 3 Genovese - Ospedale La Colletta - PO Ponente, UO di Cardiologia Riabilitativa

Ospedale Umberto I - Presidio dell'Azienda Provinciale di Lecco, UO di Cardiologia Riabilitativa

Ospedali Riuniti di Bergamo - Dipartimento Cardiovascolare, Sezione di Cardiologia Riabilitativa

Ospedale Bellaria Bologna, Modulo di Cardiologia Riabilitativa

Presidio di Riabilitazione Multifunzionale, Fisiopatologia e Riabilitazione Cardiorespiratoria

Campolongo Hospital SpA

Azienda ULSS15 PO Camposampiero, struttura di Cardiologia Riabilitativa e Valutazione Funzionale

Azienda Ospedaliera Careggi - UO Universitaria di Geriatria, Sezione di Riabilitazione Cardiaca

IRCCS Fondazione Salvatore Maugeri, Divisione di Cardiologia

Casa di Cura Villa delle Magnolie, UO di Cardiologia Riabilitativa

Ospedale Regina Margherita, UO di Riabilitazione Cardiologica

PO di Castelfranco Veneto, UO di Cardiologia - Riabilitazione Cardiologia

Azienda Ospedaliera Cannizzaro - Dipartimento Cardiovascolare, Struttura di Riabilitazione Cardiologia

AO Garibaldi, S. Luigi-Currò, Ascoli-Tommaselli, PO Ascoli-Tommaselli, Divisione di Cardiologia

Ospedale di Cecina - UO Cardiovascolare, Sezione di Riabilitazione Cardiologia

Casa di Cura Ambrosiana - Centro di Cardiologia Riabilitativa

Casa di Cura Villa Pini d'Abruzzo, Raggruppamento di Cardiologia Riabilitativa

Centro di Riabilitazione per Cardiopatico Serena Campagni, Servizio di Cardiologia

Ospedale San Sebastiano, Unità Operativa di Cardiologia

CORDIS - Unità di Riabilitazione Cardiologica Ambulatoriale

Casa di Cura Villa Pineta - UO di Cardiologia Riabilitativa

Casa di Cura Villa Garda - UO di Riabilitazione Cardiologica

AO "Bolognini” Seriate - PO Briolini - UO di Riabilitazione Cardiologica

IRCCS Fondazione Salvatore Maugeri, Divisione di Cardiologia

COF Centro Ortopedico Fisioterapico -

Ospedale al Mare Lido di Venezia - UO di Cardiologia, Servizio di Cardiologia e Riabilitazione Cardiaca

Ospedale di Lucca - Servizio di Riabilitazione Cardiologica

Centro Medico di Riabilitazione, UO di Cardiopnuemologia

Policlinico San Marco, Servizio di Cardiologia

IRCCS S. Maria Nascente Fondazione Don Gnocchi ONLUS, UO di Cardiologia Riabilitativa

Pio Albergo Trivulzio, UO Cardiologia Riabilitazione

Azienda Ospedaliera Istituti Clinici di Perfezionamento, UO di Cardiologia Riabilitativa

Ospedale Generale Provinciale, Divisione di Cardiologia

Ospedale San Polo, UO di Cardiologia - Modulo di prevenzione e riabilitazione cardiologica

USSL Asolo - PO di Montebelluna, Unità Operativa di Cardiologia

IRCCS Fondazione Salvatore Maugeri, Divisione di Cardiologia

Policlinico di Monza, UO di Cardiologia Riabilitativa

Ospedale di Mormanno - AS2 Castrovillari, UO di Medicina Interna e Cardiologia Riabilitativa

Ospedale di Motta di Livenza, Servizio di Cardiologia Riabilitativa e Preventiva

Clinic Center SpA

ASL NA1 - Presidio Sanitario Intermedio Napoli EST, UO di Cardiologia Riabilitativa

Azienda Universitaria Policlinico Federico II, Area Funzionale di Riabilitazione Cardiologica

Ospedale di Nocera Umbra ASL 3 Foligno, Servizio di Riabilitazione Cardiologica

Azienda ALSS15 Presidio Ospedaliero di Cittadella, Valutazione funzionale e riabilitazione cardiologia

Ospedale di Passirana di Rho, Divisione di Cardiologia Riabilitativa

IRCCS Fondazione Salvatore Maugeri Pavia, Divisione di Cardiologia

USL 2 dell'Umbria - Servizio di Cardiologia Diagnostica e Riabilitativa

Azienda USL 3 Pistoia - PO Pescia, UO Malattie Cardiovascolari Sezione di Riabilitazione Cardiologia

Casa di Cura Villa Serena SpA - Centro Medico di Riabilitazione Cardiologica e Polmonare

Casa di Cura Privata S. Giacomo, UO di Cardioriabilitazione

Azienda Ospedaliera Ospedale S. Carlo, UO di Cardiologia Riabilitativa e Preventiva

IRCCS “S. Maria agli Ulivi” - Fondazione Don Gnocchi ONLUS, UO di Riabilitazione Cardiorespiratoria

Azienda Ospedale Maggiore Crema - PO di Rivolta d'Adda - UO di Riabilitazione Cardiologica

Casa di Cura Santa Famiglia

PO Integrato Portuense, ASL Roma D - UO Semplice di Cardiologia Riabilitativa

Istituto Cardiovascolare Camogli

Azienda Ospedaliera Senese - UO Cardiologia Riabilitativa

Casa di Cura Villa Verde - UO di Riabilitazione Cardiologica

Casa di Cura Major - Sede dist. IRCCS Fondazione Maugeri Veruno - Divisione di Cardiologia

Ospedale Evangelico Valdese di Torino - Servizio di Cardiologia

IRCCS Fondazione Salvatore Maugeri - Divisione di Cardiologia

IMFR Udine - ASS n. 4 "Medio Friuli" - UO di Cardiologia Riabilitativa

IRCCS Fondazione Salvatore Maugeri, Divisione di Cardiologia

USL 6 Vicenza - Ospedale San Bortolo Vicenza, Dipartimento di Cardiologia

Auxilium Vitale Volterra SpA, UO di Cardiologia Riabilitativa
Località

Ala (TN)

$\operatorname{Arco}(\mathrm{TN})$

$\operatorname{Arco}(\mathrm{TN})$

Arenzano (GE

Bellano (LC)

Bergamo

Bologna

Bozzolo (MN)

Campolongo Eboli (SA)

Camposampiero (PD)

Careggi (FI)

Cassano Murge (BA)

Castel Morrone (CE)

Castelfranco Emilia (MO)

Castelfranco V.to (TV)

Catania

Catania

Cecina (LI)

Cesano Boscone (MI)

Chieti

Città di Castello (PG)

Correggio (RE)

Enna Bassa (EN)

Gaiato di Pavullo (MO)

Garda (VR)

Gazzaniga (BG)

Gussago (BS)

Lanzo Intelvi (CO)

Lido di Venezia (VE)

Lucca (LU)

Marina di Ginosa (TA)

Mestre (VE)

Milano

Milano

Milano

Mirano (VE)

Monfalcone (GO)

Montebelluna

Montescano (PV)

Monza (MI)

Mormanno (CS)

Motta di Livenza (TV)

Napoli

Napoli

Napoli

Nocera Umbra (PG)

Padova (PD)

Passirana (MI)

Pavia

Perugia (PG)

Pescia (PT)

Piossasco (TO)

Ponte dell'Olio (PC)

Potenza

Pozzolatico (FI)

Rivolta d'Adda (CR)

Roma

Roma

Ruta di Camogli (GE)

Siena

Taranto

Torino

Torino

Tradate (VA)

Udine

Veruno (NO)

Vicenza

Volterra (PI) 